\title{
The discrete time multichannel three-dimensional probability CSMA with function of monitoring based loT
}

\author{
Sheng Jie Zhou ${ }^{1}$, Hong Wei Ding ${ }^{1 *}$, Zhi Jun Yang ${ }^{1}$, Qian Lin Liu ${ }^{1}$, Meng Yao Wang ${ }^{1}$ \\ 1 Information Academic, Yunnan University, Cuihu North Road NO.2, Kunming, China \\ *dhw@163.com
}

\begin{abstract}
Currently, with the rapid development of Internet of Things (IoT), users demand more and more personalized and diversified business categories, radio spectrum increasingly tense. With the exploiting of Transmission Control Protocol/Internet Protocol (TCP / IP) protocol, network achieves the objects of the intelligent identifying, locating, tracking, monitoring and managing a network. Multichannel random access mechanism maximizes the utilization of constrained spectrum resources and allocating scarce channel resources to disparate hierarchical according to business priorities with various qualitiy of service (QoS) requirements. In this paper, we propose a MAC design combining them together in one unit system under three-dimensional probability CSMA (Carrier Sense Multiple Access) adopting the average cycle analysis method to analysis. Through the paper, precise mathematical value of system throughput is got by rigorous derivations under many circumstances. The correctness of the theory and model is demonstrated with simulation results and effectiveness illustrated. In the offline phase, simulation results verify that the proposed algorithm can improves the controllability of the system, the channel utilization, system security, and reliability of packet transmission and meet the different priorities of different QoS requirements.
\end{abstract}

Keywords: Three-dimensional probability, CSMA, multichannel, IoT, throughput.

\section{Introduction}

Things to be considered as use of the Internet expansion, the application of innovation is the core of the development of things to the user experience as the core of the innovation is the soul of the development of things. IoT (Internet of Things) is an important part of the new generation of information technology [1]. Thus, by definition, "things that material objects connected to the Internet." "Things" refers to the various information sensing equipments, such as: radio frequency identification, infrared sensors, global positioning systems, laser scanners, various other devices and the Internet combine to form a huge network. The aim is to have all the items are connected to the network to facilitate the identification and management. This has two meanings: First, the core and foundation of things is still the Internet, is based on the Internet extension and expansion of the network; second, to extend and expand its client to any goods and items of information exchange and communication ${ }^{[2]}$

The role of the MAC layer is to provide fair, reliable, efficient scheduling mechanism to allocate radio channel resources, MAC protocol performance is directly related to the performance of the wireless channel utilization and the entire network ${ }^{[3]}$. It has always been important and difficult research among scholars.

And the most common MAC layer protocol is carrier sense multiple access (CSMA) with varieties of other mechanisms.
There are many sites in the wireless communication network, so the channel resource is limited. If the site sends a message according to no law, then the probability that information packets come into the collision will be greatly increased ${ }^{[4]}$. Then if we use the CSMA (Carrier Sense Multiple Access) protocol, a site listens to the status of the channel first, and then decides whether to send a message or not ${ }^{[5]}$. With the mechanism, the probability of such a collision would be significantly reduced.

During the process of information exchange and communication, in order to achieve the objects of the intelligent identify, locate, track, monitor and manage a network, then TCP / IP (Control Protocol/Internet Protocol) come into use ${ }^{[6,7]}$.

For TCP / IP protocol, if the recipient successfully receives the data, it will return an ACK. ACK signals usually have their own fixed format, the length of size, to reply to the sender by the recipient. The format depends on which kind of the network protocol is taken ${ }^{[8]}$. When the sender receives the ACK signal, it can send the next data. If the sender does not receive a signal, then the sender may retransmit the current data package, data transfer may stop. The process is depending on the network protocol used.

Currently, with the rapid development of IoT, users demand more and more personalized and diversified business categories, radio spectrum increasingly tense. Multichannel random access protocol can maximize the utilization of 
spectrum resources and to allocate resources according to business priorities various QoS (qualitiy of service) requirements. On the future development of mobile communications, it will play an important role.

To achieve the above functions we propose the multichannel three-dimensional probability CSMA with function of monitoring (MMTDP-CSMA). The agreement not only enables the function of intelligent identify, locate, track, monitor and manage a network, but also realizes maximizing the utilization of spectrum resources and allocating resources according to business priorities various QoS requirements.

The rest of this paper is organized as follows: Section 2 describes the based protocol three-dimensional probability CSMA. Section 3 presents our MMTDP-CSMA protocol in detail. To evaluate the performance of the MMTDP-CSMA protocol, simulation setup and results are presented in Section 4. Finally, Section 5 concludes the paper.

\section{Three-dimensional probability CSMA}

The basic idea of CSMA is that before a site attempts its transmission, it needs to infer the channel condition by sensing the channel. If it infers that its transmission will upset (or be upset by) any receiver's ongoing transmissions (including its own receiver), then it defers its transmission. In addition, to prevent two sites from beginning their transmissions at the same time (given that they both sense the channel to be safe for transmission), each transmitter undergoes a random back-off countdown period before transmission .

For three-dimensional probability CSMA, there will be three random events appearing repeatedly: a packet is sent successfully ( $U$ events), transmission is done unsuccessfully namely two or more packets are sent at one time (C events), no packets need to be transmitted that channel is idle (I events), these three events are forced into: channel is idle (I events) event, channel is busy (CU events) and channel is idle following the $\mathrm{CU}$ events (CUI events); packet is sent successfully or unsuccessfully(combined $\mathrm{C}$ events with $\mathrm{U}$ event, denoted by CU event); force CU events and CUI events into B events. A cycle period is $T_{n}$. TP is transmission period. Use three-dimensional probability: P1, P2, P3 to control the period of I events, CUI events and CU events separately. At I events phase, a site send a packet at probability P1. During the CU events, the site senses channel status with probability P2. Similar to CU events, while a site is in CUI events, it senses channel status at probability $\mathrm{P} 3$.

Basic unit of the system control clock is $a$, the information packets arrived at time $a$ will transmit at the starting time of the next slot;

Channel propagation delay is $a$,the packet length is unit length and is an integral multiple of $a$;

Packets need to be sent at the first slot in the transmission period can always sense the state of channel at last moment;

During the transmission period of information packets, the phenomenon of packet collisions occur inevitably, and continues to be sent after a random time delay, it sends will not produce any adverse effects on the arrival process channel

In a cycle, the average length of time slot that information packet has been successfully sent in a cycle is:
$E(U)=E\left(U_{1}\right)+E\left(U_{2}\right)=\frac{a p_{1} G e^{-a p_{1} G}}{1-e^{-a p_{1} G}}+\left(a p_{2}+p_{3}\right) G$

Average length of $\mathrm{B}$ event is:

$E(B)=E\left(N_{B}\right) \times(1+a)=\frac{1+a}{1-e^{-\left(a p_{2}+p_{3}\right) G}}$

Where $(1+a)$ represents the length of information packet whether it transmitted successfully or not in the TP cycle.

Average length of I event is:

$$
E(I)=E\left(N_{I}\right) \times a=\frac{a}{1-e^{-a p_{1} G}}
$$

System throughput of three-dimensional probability CSMA is:

$$
S=\frac{E(U)}{E(B)+E(I)}=\frac{\frac{a p_{1} G e^{-a p_{1} G}}{1-e^{-a p_{1} G}}+\left(a p_{2}+p_{3}\right) G}{\frac{1+a}{1-e^{-\left(a p_{2}+p_{3}\right) G}}+\frac{a}{1-e^{-a p_{1} G}}}
$$

\section{MMTDP-CSMA}

With the multi-channel mechanism, there is more than one channel; we assume it owns $\mathrm{N}$ channels.

Thus considering the system having $\mathrm{N}$ channels and $\mathrm{N}$ priorities, the nodes are accessed to the channel resources randomly by the business priorities of themselves which is depicted in Fig. 2.

Assume that the priority sequence is arranged from low to high as priority 1 , priority $2 \ldots$ priority $\mathrm{N}$. The service with priority $i$ occupies channel 1 to $i(i=1,2 \ldots \mathrm{N})$, that is the service with priority 1 occupies channel 1 , priority 2 occupies channel 1 and channel 2 , and the service with priority $\mathrm{N}$ occupies channel 1 to channel $\mathrm{N}$.

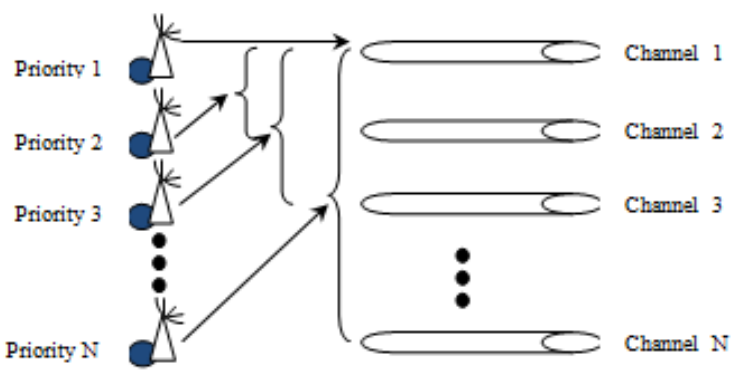

Fig. 1. Structure of multichannel mechanism with $\mathrm{N}$ channels

For a single channel, assuming channel $i$, under the MMTDP-CSMA mode, packet transmission and retransmission phase is the same as three-dimensional probability CSMA. Unlike the above B events scenarios, when during the transmission interval, the time is added to $(1+3 a)$ while the previous is $(1+a)$, the addition time slot $2 a$ is exploited to transmit ACK or NCK information.

Before analysis, do assumptions as before while adding one more: the access method of number $i(i=1,2, \ldots, N)$ channel is timeslot three-dimensional probability CSMA, and the arrival process of number $i$ channel satisfy the Poisson 
process whose independent parameter is $G_{i}$, each arrival process on the channel is independent of each other.

For channel $i$, in a cycle, the average length of time slot that information packet has been successfully sent in a cycle is:

$$
E\left(U_{i}\right)=E\left(U_{1, i}\right)+E\left(U_{2, i}\right)=\frac{a p_{1} G_{i} e^{-a p_{1} G_{i}}}{1-e^{-a p_{1} G_{i}}}+\left(3 a p_{3}+p_{2}\right) G_{i}
$$

(5)

Average length of B event in channel ${ }^{i}$ is:

$$
E\left(B_{i}\right)=E\left(N_{B_{i}}\right) \times(1+3 a)=\frac{1+3 a}{1-e^{-\left(3 a p_{3}+p_{2}\right) G_{i}}}
$$

(6)

Where $(1+3 a)$ represents the length of information packet whether it transmitted successfully or not of channel $i$ in the TP cycle.

Average length of I event in channel $i_{\text {is: }}$ :

$$
E\left(I_{i}\right)=E\left(N_{I_{i}}\right) \times a=\frac{a}{1-e^{-a p_{1} G_{i}}}
$$

For channel $i$, system throughput of MMTDP-CSMA is:

$$
S_{i}^{\prime}=\frac{E\left(U_{i}\right)}{E\left(B_{i}\right)+E\left(I_{i}\right)}=\frac{\frac{a p_{1} G_{i} e^{-a p_{1} G_{i}}}{1-e^{-a p_{1} G_{i}}}+\left(3 a p_{3}+p_{2}\right) G_{i}}{\frac{1+3 a}{1-e^{-\left(3 a p_{3}+p_{2}\right) G_{i}}}+\frac{a}{1-e^{-a p_{1} G_{i}}}}
$$

Basing on the above analysis and computational formula of the systemic throughput.

systemic throughput of MMTDP-CSMA is:

$$
S=N S_{i}^{\prime}
$$

Assuming that the length of information packet sent by the business with priority $l$ successfully in average cycle period of channel $i_{\text {is: }} E\left(U_{i}^{(p l)}\right)(i \leq l)$

Then according to the above analysis, we can get the throughput of the MMTDP-CSMA with the priority $l$ :

$$
S_{p l}=\left(\sum_{i=1}^{l} \frac{1}{N-i+1}\right) S_{i}^{\prime}
$$

\section{Simulation results and analysis}

From the above analysis, the expression of the system throughput under the discrete time three-dimensional probability CSMA protocol with multichannel mechanism is got. With the simulation tool-MATLAB R2010a, the simulation results are shown in Figure 4 to Figure 7. If not specified $a=0.01$.

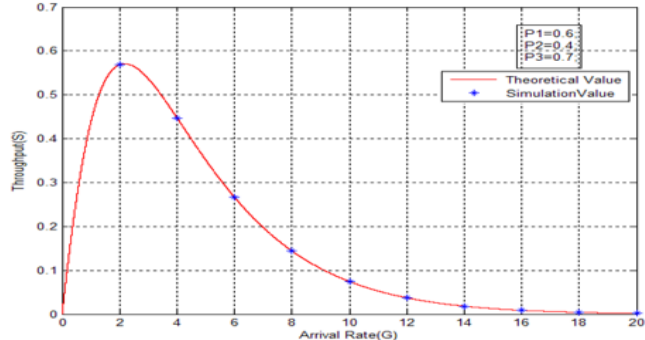

Fig. 2. The throughput of the new protocol for channel $i$

As it can be seen from the Fig. 2, the simulation values of system throughput under the new protocol are consistent with the theoretical ones. At the beginning, when the arrival rate increases, the system throughput also increased; then throughput reaches its maximum under the condition. Finally, with the increasing amount of the arrival rate, the system throughput decreases. The simulation values of system throughput under the new protocol are consistent with the theoretical ones.

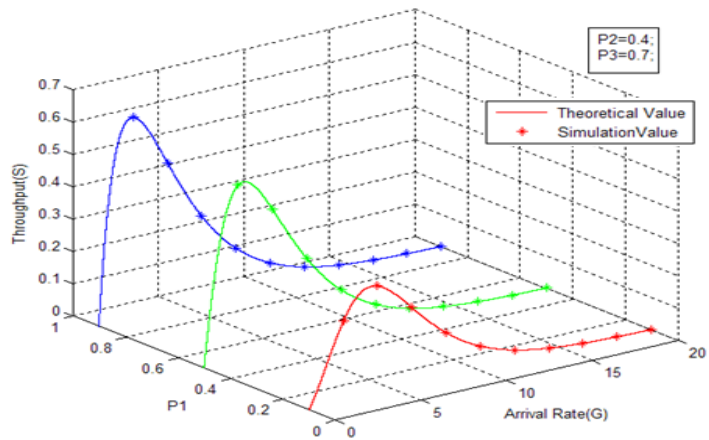

Fig. 3. The throughput of the new protocol with variable parameter $\mathrm{P} 1$

Can be seen from the Fig. 3, the simulation values of system throughput are consistent with the theoretical ones. We can find that with the $P 1$ increases, the system throughput is increases too. This is laying that when the arrival rate is small, if the probability of arrival information sent is too small at the I events, the channel resource is not fully utilized. Thus, if we increase the probability of the arrival information transmitted at this time, we can improve the efficiency of channel resources and increase the value of the system throughput.

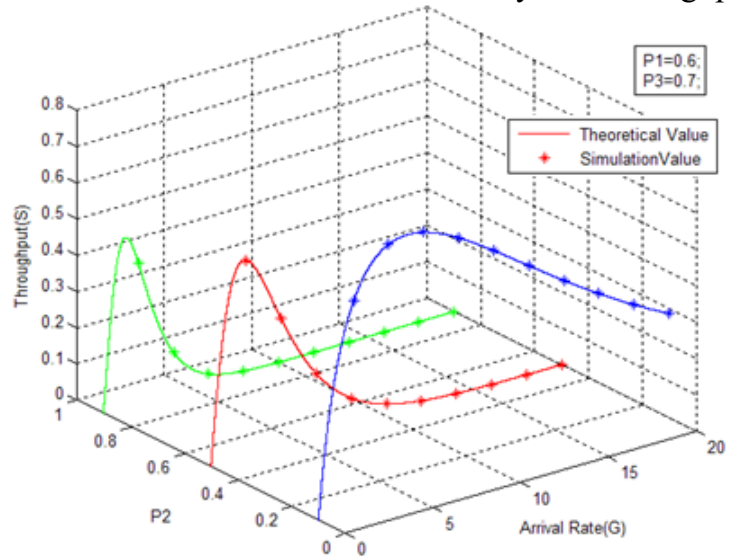

Fig. 4. The throughput of the new protocol with variable parameter $\mathrm{P} 2$

In the Fig. 4, the simulation values of system throughput under the new protocol are consistent with the theoretical ones. As can be understood from the figure, when $P 2$ becoming bigger, the throughput will decrease; because when the 
channel is busy sending the packet, the more new arrival information packets to send at the $\mathrm{CU}$ events the more collisions will be.

So we can change variable $P 3$ to control the process of the CUI events. Not only this, we can change the variable of $P 1$, $P 2$ and $P 3$ at the same time to get value of the system throughput needed.

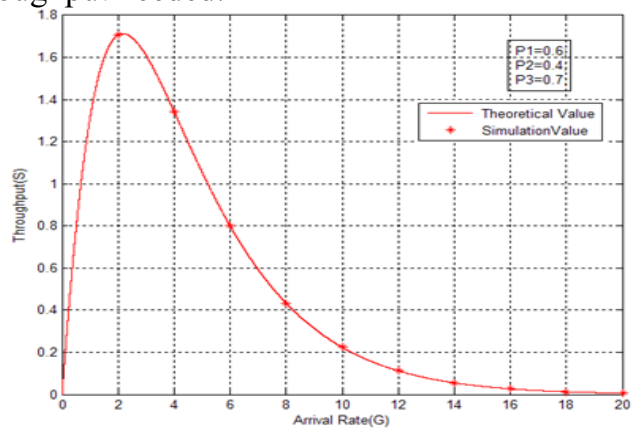

Fig. 5. The throughput of the new protocol with 3 channels

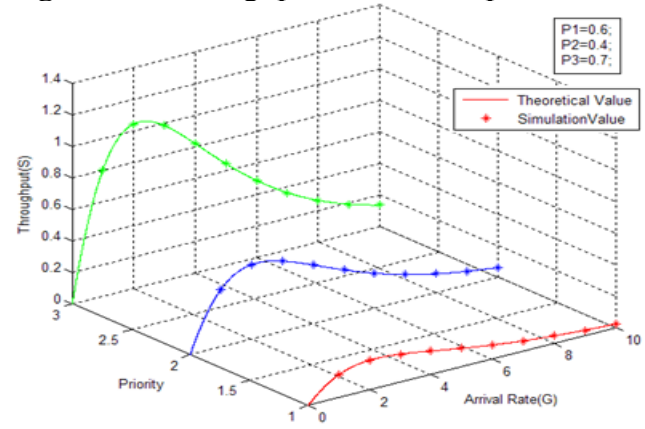

Fig.6. The comparison of system throughput owning 3 channels with different priorities

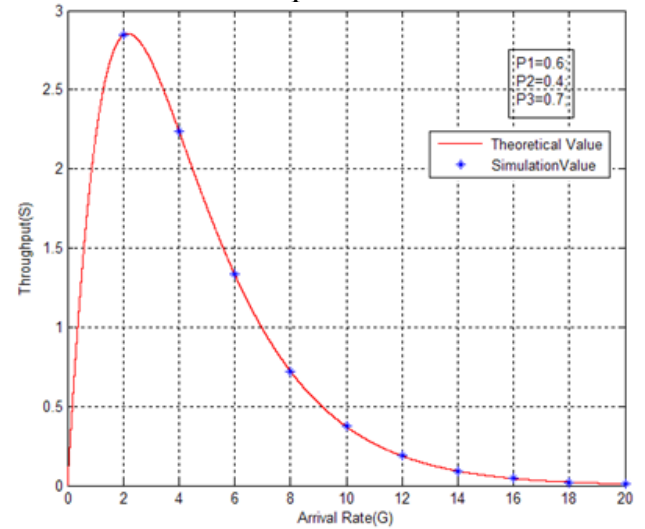

Fig. 7. The throughput of the new protocol with 5 channels

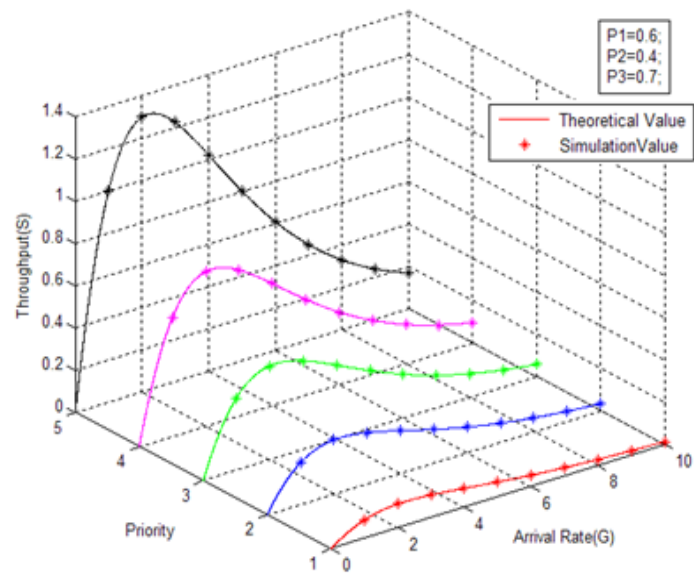

Fig. 8. The comparison of system throughput owning 5 channels with different priorities

As can be understood from Fig. 5 to Fig. 8, the simulation values of system throughput under the new protocol are consistent with the theoretical ones. With the total number of channels increases, the value of the new protocol's total system throughput will increases; the channel resources can distribute to every channel according to their priority according to their own priority separately; when the priority is higher, the corresponding single channel will get more network resources than the lower priorities; thus the value of throughput with higher priority channel is bigger than others with lower priorities. With the multi-channel mechanism, the network resources utilization has been improved significantly.

\section{Conclusions}

With radio spectrum increasingly tense, to satisfy the users' demand of more personalized and diversified business categories. To realizing the functions the intelligent identify, locate, track, monitor and manage a network. Using the average cycle analysis method to analysis the proposed protocol of the discrete time multichannel three-dimensional probability CSMA protocol with function of monitoring, realizing the functions above at the same time; during the paper, through the vigorous deviations obtains the accurate mathematical expressions of the system throughput. The correctness of the theory and model is demonstrated with the simulation tool-MATLAB and its effectiveness is illustrated.

\section{References}

[1] Yuhong, Z., Wei, L.: 'Modeling and energy consumption evaluation of a stochastic wireless sensor network ', EURASIP Journal on Wireless Communications and Networking, 2012, 4, (1), pp.79-82

[2] Dongfeng, Z.: 'Study on A New Method for Continuous-time Systems of Random Access Channel', Journal of Electronics, 1999, 21, (1), pp.37-41

[3] Hongwei, D., Yingying, G., Yifan, Z., et al.: 'Sensor Lett',2015, 13, pp.143-146

[4] E. A, B.: 'Towards robust distributed systems ACM New Method for the Slotted Access Channel, Journal of Electronics, 1997, 19, (6), pp.814-819

[5] Wenmiao, S., Yanming, L.: 'Research on S-MAC protocol for WSN', IEEE Wireless Communications, 2008, pp.779-782

[6] Dongfeng, Z., Bihai, L.., Sumin, Z.: 'Study on a New Method for the Slotted Access Channel', Journal of Electronics, 1997, 19, (6), pp.814-819

[7] Dongfeng, Z.: 'Study on the Average Cycle Method for Slotted Multiple-Access Communications', Journal of China Institute of Communications, 1999, 20, (8), pp.80-85

[8] Dongfeng, Z.: 'Study on A New Method for the Slotted Access Channel', Journal of Electronics,1997, 19, (6), pp.814819 